\title{
Healthcare Simulation: A Psychological Tool to Induce Stress
}

\author{
Natasha Taylor ${ }^{\dagger}$
}

\begin{abstract}
Background

Simulation or scenario learning is an integral part of student paramedic development and, despite the increasing amount of paramedic research, very little is known about how the students and tutors experience it. Current literature regards simulation as invaluable without exploring why this may be the case and this study aimed to address this.
\end{abstract}

\section{Methods and findings}

This compressed time mode ethnographic approach incorporated data from student paramedics during and immediately after the simulation learning event and tutor views of facilitating the simulation experience.

\section{Conclusions}

This study exposed how student paramedics find the simulation process anxiety provoking and explored the possible psychobiological reasons for this.

\section{Keywords}

Paramedic development; Psychobiological; Ethnographic approach

\section{Introduction}

In the United Kingdom there are fifteen National Health Service (NHS) ambulance services; these are split into geographic areas based on county, area or country. In these fifteen ambulance services, there are many different ways to achieve paramedic status. These pathways can be vocational, higher education or a mixture of both but all share similar development tools. Regardless of which development pathway(s) an ambulance service makes use of, simulations are used as part of an entire course strategy. This includes practical and theoretical learning and assessments applied both formatively and summatively. Simulation typically makes up nearly one-half of total course time and some simulation assessment must be passed successfully to progress.

Stress and paramedicine are interlinked. Most people would assume that paramedics work in a stressful environment attending to patients in (sometimes) horrific situations and it could be accepted that this is true. Media portrayals of paramedicine seem to depict major incidents and life-threatening emergencies continuously throughout every shift. This is not the case and the average shift consists of periods of relative calm, driving and attending routine incidents, interspersed with intense short periods of time with undoubted potential stressors. Stress may have different meanings in different situations and therefore it is important to explore the nature of stress and its relationship with anxiety and simulation. The term stress has its origins 
in engineering and can be defined as an external threat or tension from a stressor [1]. These stressors can cause the fight-or-flight effect of the sympathetic adrenomedullary system, causing release of adrenaline and noradrenaline and it is this uncomfortable feeling that is vital to continued human existence. These increases in involuntary processes such as heart rate and respiratory rate, along with other symptoms such as peripheral vasoconstriction and increased blood flow to the voluntary muscles causes some of the unpleasant side effects of this response. Perhaps a more disturbing and in the long term more dangerous hormone of stress is cortisol, more often released during acute psychological stressors (such as undertaking a simulation). Continued stress causes hormonal release and potential unpleasant and relatively harmful side effects. There is some evidence to suggest that constant or repeated stress can permanently damage the hippocampus leading to memory loss [2]. This takes into account the concept of threat versus challenge, threat inducing a full stress response and challenge being merely heightened sympathetic arousal to obtain a goal. The concept of challenge and the processes that govern it, although unpleasant, are vital in helping us achieve our goals. It sharpens reflexes, ensures a heightened blood flow to the brain and generally makes us perform better. As discussed, anxiety is a cognitive state that is part of the overall stress mechanisms and it is anxiety that (when related to simulation) may cause such dislike of this learning tool.

\section{Methods}

This study used an ethnographic approach to methodology and methods. Ethnography, as a product, should allow for the study of social occurrences and the analysis of the meaning of these phenomena; in this case, the social occurrences that occur in an ambulance education setting. However, it may be argued that ethnography is not only a product but also a process [3]. Ethnographic data analysis generally involves the interpretation of meanings in a social setting and functions of human actions and may be carried out in a wide range of settings with almost limitless theoretical perspectives. Any place where people come together, such as a work area or residence, anywhere where interaction occurs may be used [4]; therefore, it was appropriate in to use this methodology in this context, for this research. The methodological principle of cultural relativism may have helped to make sense of the social interactions that were observed. Cultural relativism is the principle of understanding a culture from within, in its own terms and it proved difficult at times to maintain this stance as an 'insider' rather than an external researcher. This principle necessitated understanding the culture studied in its own terms, this is especially important when the researcher is familiar with the culture studied. The tacit assumptions were acknowledged and, if possible, suspended. One of the seemingly widespread fears for ethnographers is the risk of 'going native' $[5,6]$. Although this seems a moot point, being a 'native' of ambulance education already, some consideration was given to this issue but the use of a reflexive ethnography lends itself very well to this issue. The concept of becoming an outsider wasn't applicable in the research and any attempt to do otherwise would have been merely to adhere to some accepted norms in ethnographic research. In this research, the concept of already being an insider was embraced, as it allowed the data to make sense in a way that wouldn't have been possible without pre-existing thoughts, feelings and even prejudices. This concept is gaining credibility in research and there is some acceptance of 'native' research as valid [7].

The selection of participants was based on the courses running at the time of the data collection period in the three centres used; all participants had to be students on an ambulance development pathway. Some participants were on their initial development, not having been operational at all, some were part way through development, having spent a great deal of time in an accident and emergency setting. The initial call for students to take part was via a classroom discussion and one page letter given to all student paramedics on a development course, calling for participants. Out of a total of approximately fifty students who would have been eligible, fourteen students agreed to be observed and for a subsequent interview to be recorded. All of the students that took part in this study completed a participant consent form outlining their right to anonymity and their right to withdraw at any stage. Confidentiality was promised and upheld and this study received approval from University and organization ethics committees. British Educational Research Association (2014) guidelines were adhered to throughout the research. Therefore, the final number of participants were fourteen $(\mathrm{N}=$ 14) students, who were observed individually 
during simulation exercises and then interviewed immediately after the simulation was concluded. All names were anonomised and placed in alphabet order for ease of reading.

The research design was not closely structured at the start and themes came from the data rather than having set theories to explore. This idea, that the data collected may cause themes to emerge that can then be analysed is not new or special to ethnography. The 'grounding' of theory in data has been used effectively in this type of research for decades, although there are serious differences between 'ethnographers' and 'grounded theorists' depending on their philosophical and ontological assumptions; Glaser and Strauss being the first to formally acknowledge the use of this methodological tool [8]. It is also suggested that this grounded theory is a particularly appropriate methodology for areas of study where established theory or data is missing; this method (and methodology) generates rather than verifies theory. The data obtained were interpreted to extract meaning and consequence rather than any formal statistical analysis. The lack of numerical or objective contrasts must be outweighed by acknowledging ethnographic tools such as triangulation. Here too, a more participatory approach to data collection was used and if participants were willing, data analysis and presentation and verification allowed for a more ethical design as well as a more robust means of allowing multiple perspectives and views to influence the study.

\section{Results}

I think it's because for me, I'm nervous about it because in my head I'm trying to remember everything and ... it's going all gobbledegook in your head and you're trying to remember the structure and I think you get flustered and if something's thrown out different, it kind of gets you in a flap and you're trying to get yourself back together and you've got to stop, get yourself back and that's what I've been pretty much doing the whole time.

(Katie)

Anxiety can cause a number of physiological effects that may be seen and certainly can be felt. As discussed, anxiety is a cognition state as part of the wider set of behaviour and physiological responses of (and to) stress. Apprehension, tension and worry are the feelings most associated with anxiety but in this study there is a clear line between anxiety as a by-product of simulation learning and anxiety as a set of symptoms that affect ability to function. This generalized anxiety disorder may be considered as excessive or persistent and not the immediately occurring and subsiding anxiety associated with simulation learning. This is the definition meant when referred to anxiety in this study. In all of the simulations observed, although it was clear that students were anxious, they were all able to complete the scenario, however abhorrent the anxiety effects may have been.

When discussed with students and tutors alike, both accepted that ambulance work could be stressful at times. Charles, when asked if he felt paramedicine has moments of stress stated emphatically "God yes, from what I've seen, there are some really bad jobs, car crashes, that sort of thing with major injuries. Of course that's stressful". Yale, a very experienced paramedic and tutor noted, "[the job] really is full on at times, you go to some horrible [jobs], it just takes it out of you". Certainly in ambulance work, it is generally acknowledged that paramedics suffer stress from what may be considered stressful emergency calls and this has been studied for many years few authors stated [9-11], in common with virtually all paramedic stress evidence, that mental and physical stress required better training to detect and treat but did not consider stress whilst training. There is no doubt that emergency ambulance work is (on occasions) stressful and adrenaline response can be felt and observed. However, the link between: a) the stress of paramedicine whilst operational and b) the stress of simulated paramedicine in the education setting have many factors in common. What is certain is that the simulation events observed caused anxiety for the overwhelming majority of student paramedics talked to.

"[Alan] looks ill, ashen ... he has sweat rings on his shirt" was noted in the study field journal and Katie identified that during simulation "my heart's going because of my nerves". These descriptions accurately reflect the physiological responses of acute stress. The pale skin seen in anxiety and increased sweat secretion allows for the radiation of heat away from the skin in moments of potential immediate physical work. The increased cardiac contractility and rate ensures the potential rapid physical need is fed by an increase in blood pushed around the body. An increase occurs in speed of heartbeat and in the amount of blood each ventricular contraction drives into the system. Also noted was "[John] can't keep still, twitchy, shaky" 
during the simulation. The increased blood flow to the limb muscles allows the legs and arms to be stronger and faster but if no exercise is taken, can cause the characteristic twitchiness or trembling of the arms and legs. This was seen most obviously by students pacing prior to the simulation and when questioned about this, Dan agreed, "It's probably just fight-or-flight". Dan identified his own physiological response to the simulation event, using the lay term for the response. During the adrenergic response, the gastrointestinal processes are of no use and may cause blood to be required that could be better used elsewhere. There is a peculiar voiding and maintaining pattern that means that much of the gastrointestinal tract slows whilst the parts most open to the outside world tend to void, hence the urge to be sick and visit the lavatory in moments of stress whilst feeling nauseous due to the slowing of gut motility. This was clearly articulated by some of the student paramedics, Michael stated "[doing simulations makes me] want to have a crap" and Natalie insisted that she "always feel[s] sick when I'm waiting to go in [to a simulation]". However, symptoms of anxiety have been experienced by most people when faced with moments of stress and these are entirely natural and necessary processes however much discomfort they cause. All of the physiological responses are to reduce peripheral blood flow and increase shunting of oxygen and nutrient-rich blood to the central organs but it is the prolonged and continuous anxiety response that is most harmful. Therefore, as well as the physiological response seen and felt, the student paramedic was also able to articulate this concept of anxiety during the simulation event [12].

The initial interview question, when talking to the student paramedic after their participant observation ended, was the same, "how was that scenario for you"? All of the simulations observed were considered learning scenarios rather than assessment scenarios; although this distinction may not be quite so clear. This was a necessarily open question that the student could have interpreted in many ways but it was surprising that all, except one participant, indicated that they felt nervous or apprehensive during the simulation. Not one student discussed their clinical performance during the simulation and all stated a clear discomfort in the process. There is some evidence regarding stress in ambulance simulation but this is merely evaluating stress based on ambulance work rather than analyzing the stress of the simulation itself. LeBlanc [13], found inherent stressors in the examination process but there was no examination of these ambulance education simulation stressors. It is even acknowledged in one paramedic textbook that simulation is not a pleasant experience for the student, "run critiques [simulations] can be great learning experiences, don't consider them to be punitive" [14]; this is said without elucidating further. It almost seems to be an unwritten rule in paramedicine that simulation is a necessary baptism of fire to help prepare for the undoubted stress of the job of a paramedic.

Frank suggested that "[the simulation is] ok, I can't imagine being let out there [on the road] without having done it; I wouldn't feel very confident about my treatment" and it must be accepted that there is some literature that has suggested that simulation learning increases confidence slightly $[15,16]$. This echoed data collected for this study; Frank could not imagine being operational without having undertaken simulation. There is also some evidence that some paramedics evaluate the use of some simulations highly [17], although this study appears to be an evaluation of simulation equipment rather than the process of simulation learning or assessment itself. Prentice [18], suggested that simulation was highly effective as a teaching strategy based on participant feedback; staff at a community hospital felt that simulation was a useful way to learn new skills. This is seen in the overall commitment to simulation seen by the student paramedics, who felt that scenarios were fundamental to paramedic education. Another question asked of all participants (both students and tutors) was "would you remove simulation [from the course you are currently undertaking]"? All student paramedics and tutors said no, that simulation should not be taken out of the course, some being more insistent than others. Bryony emphatically stated that "absolutely no way [should simulation be removed from the course]". Natalie argued, "I hate [simulation] but you can't not do it"; when asked why, her eyes widened and she shook her head and was almost speechless that the interviewer had suggested such a ridiculous proposal.

\section{Discussion}

It is certain that all of the student paramedics talked to as part of this study not only showed the physical response of anxiety but also articulated this (without prompt) during interview. As already discussed, all of the simulations were 
learning scenarios rather than assessment scenarios and therefore the examination anxiety of simulation should not have been relevant. Therefore, although the student paramedic feels anxiety about the simulation process, the problem may be more complex than merely suggesting possible anxiolytics. Suggesting strategies to alleviate anxiety without exploring the root causes may be ultimately pointless. On the other hand, although the physiological effects of stress can be harmful, stressful simulation echoing the stressful nature of paramedicine may help to prepare the student for life as a paramedic.

There is even a possibility that the very nature of simulation is inherently stressful; scenarios seem to mirror the standard psychological test to induce stress, the Trier Social Stress Test [19]. The four defining aspects of this test can be related directly to student paramedic simulation events as already highlighted in this study, specifically:

i. Public speaking (the verbalisation by the student),

ii. Mental arithmetic (the calculation of drug dosages, weight and equipment sizing),

iii. Having an audience (the tutor, simulated patient and crewmate),

iv. Anticipatory period (the student waiting to be called into the simulation room) [19].

It is interesting to note that the test to induce stress was originally intended for individuals; however, similar results were found in group situations [20]. Therefore, simulation may be inherently stressful and the stress of the scenario may be a useful tool in helping to prepare the student paramedic for a sporadically stressful operational career. However, examining possible causes of simulation stress may identify where changes may be made and clear stressors emerged from the data.

Therefore, the position of this paper is that student paramedics find simulation learning anxiety provoking; a theory borne out of both interview data and observation of physiological alterations found in students. Perhaps the most revealing theory to emerge from these data is that the student paramedic views a strong (but in many ways not acknowledged) association between simulation learning and simulation assessment. This association is clear in the student paramedic mind, they regard all simulation as assessed in some way and that may be true. There appears to be no obviously discernable difference between simulation learning and simulation assessment in the opinion of the student. The simulation learning experience is not the simulation assessment experience but students invariably link the two. This may be for very good reasons educators and the ambulance service does nothing to separate these two entities. However, perhaps the student needs to go through the process to ensure they are fit to work in the undoubtedly stressful world of emergency medicine. Anxiety is a common and essential physiological process and paramedicine a stressful profession. However, whether this needs be transferred directly experienced through paramedic education is debatable. This seems unnecessarily cruel, with no evidence to suggest its usefulness. Reducing anxiety is not necessarily about increasing pass rate or examination scores (the usual markers in literature), it is about ensuring the student receives the best possible learning experience. Therefore, it could be suggested that simulation should be made less stressful. Putting the student through times of stress does not seem the wisest way to get them to prove their worth; it may merely sour the experience for all future simulation events.

\section{References}

1. Noy S. The traumatic process: conceptualization and treatment. Prehosp. Disaster. Med 19(1), 37-45. (2004).

2. Kemeny ME. The psychobiology of stress. Curr. Dir. Psychol. Sci 12(4), 124-129 (2003).

3. Wolcott HF. Making a study "more ethnographic". In: J Van Maanen J, editor. Representation in ethnography (79-111). Thousand Oaks, CA: Sage Publications (1995).

4. Boyle JS. Styles of ethnography. In: Morse JM, editor. Critical issues in qualitative research methods. Newbury Park, CA: Sage 137-162
(1994).

5. Hammersley M. What's wrong with ethnography: methodological explorations? London: Routledge (1992).

6. Clough PT. Comments on setting criteria for experimental writing. Qual. Inq 6(2), 278-291 (2000).

7. Kanuha VK. "Being" native versus "going native": conducting social work research as an insider. Soc. Work 45(5), 439-447 (2000).

8. Glaser B, Holton J. Remodelling grounded theory. The Grounded Theory Review. 4(1), 1-24 (2004).
9. Elling R. Stress as related to the EMT-P. EMT J 4(4), 32-34 (1980).

10. Hammer JS, Matthews JJ, Lyons JS, et al. Occupational stress within the paramedic profession: an initial report of stress levels compared to hospital employees. Ann. Emerg. Med 15(5), 536-539 (1986).

11. James A. Perceptions of stress in British ambulance personnel. Work. Stress 2(4), 319326 (1988).

12. Okada N, Ishii N, Nakata M, et al. Occupational stress among Japanese emergency medical technicians: Hyogo Prefecture. Prehosp. 


\section{Research Natasha Taylor}

Disaster. Med 20(2), 115-121 (2005).

13. LeBlanc V, MacDonald R, McArthur B, et al. Paramedic performance in calculating drug dosages. Prehosp. Emerg. Care 9, 439-444 (2005)

14. Sanders M. Mosby's paramedic textbook. St Louis, MI: Mosby; (2001).

15. Alinier G, Hunt B, Gordon R, et al. Effectiveness of intermediate-fidelity simulation training technology in undergraduate nursing education. J. Adv. Nurs 54(3), 359-369 (2006).
16. Gordon JG, Buckley T. The effect of highfidelity simulation training on medicalsurgical graduate nurses' perceived ability to respond to patient clinical emergencies. J. Contin. Educ. Nurs 40(11), 491-498 (2009)

17. Wyatt A, Archer F, Fallows B. Use of simulators in teaching and learning: paramedics' evaluation of a patient simulator. J. Emerg. Prim. Health. Care 5(2), 1-16 (2007).

18. Prentice D, Taplay K, Horsley E, et al. Interprofessional simulation: an effective training experience for health care professionals working in community hospitals. Clin. Simul. Nurs 7(2), 61-67 (2011).

19. Kirschbaum C, Pirke KM, Hellhamme DH. The 'Trier Social Stress Test' - a tool for investigating psychobiological stress response in a laboratory setting. Neuropsychobiology 28(1-2), 76-81 (1993).

20. Von Dawans B. Kirschbaum C. Heinrichs M. The Trier Social Stress Test for Groups (TSST-G): A new research tool for controlled simultaneous social stress exposure in a group format. Psychoneuroendocrinology. $201136(4), 514-522$. 